\title{
ON THE EIGENMODES AND EIGENFREQUENCIES OF LOW-DIMENSIONAL DEGENERATED CARBON STRUCTURES: OBTAINING NATURAL FREQUENCIES OF IDEAL AND STRUCTURALLY DEFECTED SYSTEMS
}

\author{
SAdegh Imani YengeJeH \\ Centre for Clean Environment and Energy, School of Environment and Science, Griffith University, Australia \\ e-mail: imani.sd@gmail.com
}

Seyedeh Alieh Kazemi

The University of Birjand, Department of Mechanical Engineering, Birjand, Iran

e-mail: sa.kazemi83@gmail.com

ANDREAS ÖCHSNER

Esslingen University of Applied Sciences, Faculty of Mechanical Engineering, Esslingen, Germany

e-mail: andreas.oechsner@gmail.com

\begin{abstract}
We concentrated on evaluating the vibrational response of ideal and defected degenerated carbon nanostructures under the influence of different boundary conditions. In addition, an attempt has been made to investigate the relative deviation of the natural frequency of imperfect systems and to study the effect of defected regions on vibrational stability of the particles. It has been found that a single and pinhole vacancy defect have the least and the most impact on the natural frequency of nanostructures. Furthermore, the effect of CNT diameter on natural frequencies of low-dimensional systems has also been investigated in this research.
\end{abstract}

Keywords: carbon nanostructures, finite element method, vibrational property, vacancy defect

\section{Introduction}

In the recent decades significant attention has been paid to exploration of characteristics of low-dimensional carbon structures including carbon nanotubes (CNTs) and their degenerated nanostructures (Iijima, 1991; Chandra and Namilae, 2006; Yao et al., 2008, Imani Yengejeh et al., 2014a,b, 2015a,b). Such nano-configurations possess outstanding physical, thermal and mechanical properties and, therefore, seem to be effectively applicable in the industry domain from manufacturing to aerospace engineering (Ruoff and Lorents, 1995; Lu, 1997; Imani Yengejeh et al., 2015a,b). Innumerable studies have been conducted in order to find and explore characteristics of CNTs. Prediction of tensile strength (of up to $63 \mathrm{GPa}$ ) and Young's modulus (of nearly $1 \mathrm{TPa}$ ) of CNTs was the most significant aim of those investigations (Tserpes and Papanikos, 2007; Kuang and He, 2009; Imani Yengejeh et al., 2014b; Imani Yengejeh and Öchsner, 2015). Basically, there are two major categories for such studies: computational and experimental approaches. Continuum mechanics techniques such as the finite element method (FEM) and molecular dynamics (MD) simulations have been the most highlighted computational approaches to explore the characteristics of nanostructures (Mylvaganam et al., 2006, Hollerer and Celigoj, 2013). Despite remarkable exploration on predicting the behavior of CNTs, far more concentration is required to evaluate the mechanical behavior of CNTs and other types of low-dimensional particles such as nanocones, junction hybrids, fullerenes, etc. In the following, the 
most recent studies concerning the evaluation of vibrational property of carbon nanostructures are presented.

Bogush et al. (2017) developed an approach to study molecular vibrations for symmetrical systems. Their proposed method was applied to investigate vibrational characterization of the fullerene molecule C60 and to study a relative comparison of theoretical results and experimental data. Their study was mainly focusing on symmetrical models, and less attention was paid to asymmetric structures. Following this, Mohammadian et al. (2017) explored the vibrational response of linearly and angle-joined CNTs applying a molecular mechanics approach. They divided their investigation into two main categories. First, the influence of the junction region formation on natural frequencies of ideal hybrids was examined. Afterwards, the impact of some defects on the vibrational behavior of those models was investigated. It was noted that the frequencies and mode shapes were comparatively influenced by changing the location of the connecting region. Furthermore, it was concluded that the frequency shift of the defective configurations with lower aspect ratios was more influenced by the degree of imperfections. Although their research seemed to be effectively covering the behavior of defective low-dimensional tubes, the impact of applied impurities on the specific regions of the hybrids was not fully addressed in all details. Then, Ardeshana et al. (2017) suggested a novel method to explore vibrational characteristics of carbon nanocones. Also, their analysis exhibited the impact of varied model lengths on natural frequencies. Based on their findings, it was noted that increasing side length of a nanocone with a constant apex angle results in a significant decline in the fundamental frequency of the models. They also suggested that smaller lengths of nanocones are more likely to be applicable as they exhibit remarkable variation in the fundamental frequencies. Despite the comparatively comprehensive investigation, the behavior of imperfect low-dimensional particles was not discussed in their study.

Undoubtedly, there are some significant gaps in this research area. The objectives of this study is to explore and to predict the vibrational response of symmetric and asymmetric carbon nano-configurations in their ideal and defective forms, namely linearly- and angle-joined CNTs, open-tip carbon nanocone, open-end and capped CNTs, and fullerenes. These low-dimensional structures possess unique properties and are applicable to the experimental area. Due to holding such outstanding characteristics, prediction of their mechanical response is effectively essential in the nanotechnology scope (Imani Yengejeh et al., 2016). There are several challenges in the current paper, namely different boundary conditions, application of certain amount impurities to a particular region of the models, and evaluation and comparison of the natural frequencies of defect-free and manipulated low-dimensional configurations using FEM. The results of this research is most likely to be reliable since the majority of nanostructures found in reality are exposed to atomic and structural imperfection, and their mechanical characteristics will definitely be affected by such defects. Therefore, the proposed study will explore the possible property of imperfect degenerated nanostructures in detail.

\section{Materials and approaches}

The basic configuration of low-dimensional structures including homogeneous CNT, linearlyand angle-joined CNTs, nanocones, cylindrical fullerenes and bucky-balls can be imagined as pseudo two-dimensional (2D) single-layer sheets of graphene, as shown in Fig. 1.

Overall, seven different nanostructures have been modeled within this study, namely homogeneous CNT, one-side capped CNT, two-side capped CNT, open-tip nanocone, linearly- and angle-joined CNT, and bucky-ball. Furthermore, three different boundary conditions have been considered in simulations, i.e. free-free, fix-free, and fix-fix boundary conditions. The aim of considering numerous models and various boundary conditions is to evaluate the behavior of 


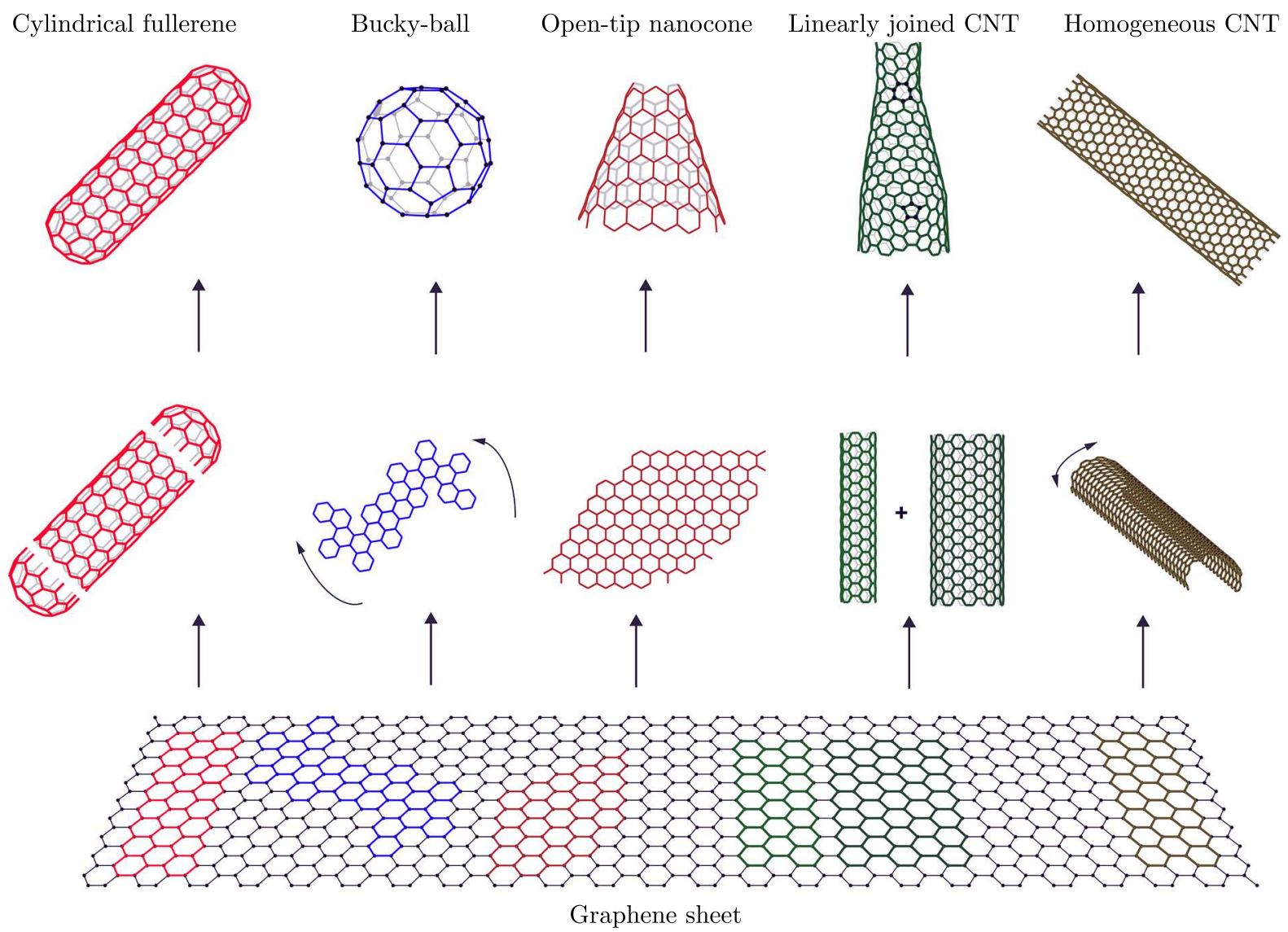

Fig. 1. Single-layer graphene sheet as carbon precursor to cylindrical fullerene, bucky-ball, open-tip nanocone, linearly-joined CNT and homogeneous CNT

different low-dimensional configurations under different boundary conditions, since they may have a significant response in different circumstances. Following the modeling and simulation of the ideal nanostructures, some common vacant sites, i.e. mono-, di-, tri-, and pinhole vacancy defects have been introduced to the perfect models to evaluate the vibrational response of low-dimensional configurations which are closer to the ones found in reality. Those mentioned atomic impurities are illustrated in Fig. 2.

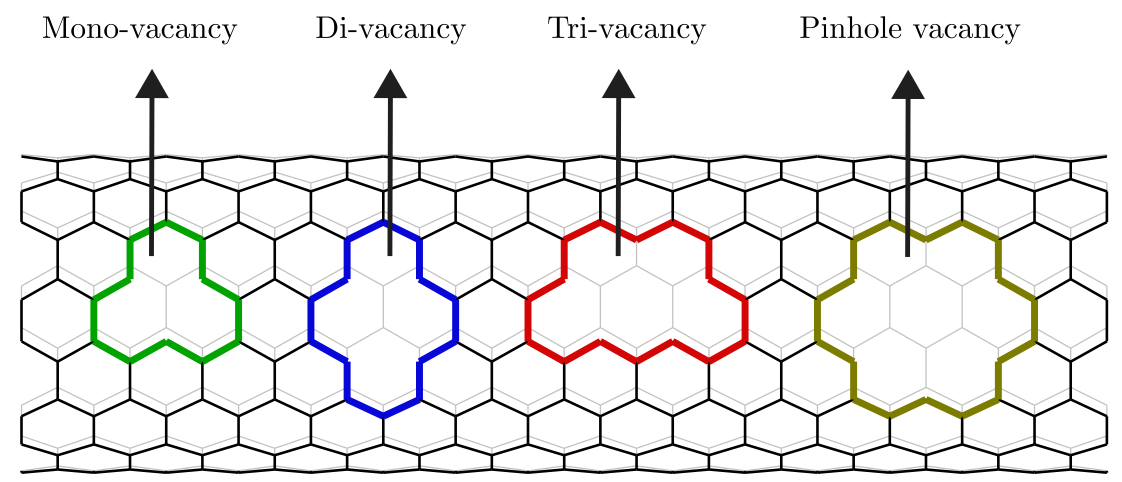

Fig. 2. Some common structural defects such as mono-, di-, tri- and pinhole vacancy introduced into configuration of the studied CNT systems

The modeling and simulation procedure is carried out in three major steps. Firstly, the spatial coordinates and connectivities of all nanostructures are imported to a commercial FE package (MSC Marc) and the first natural frequency of ideal models is obtained. Next, some of 
the most common vacant sites are introduced to the defect-free configurations. These impurities are applied to the perfect models via a custom code in MATLAB to the original models and with the same impurity percentage (1\%). In the final step, the fundamental frequency of defected structures is acquired and compared to those with perfect configurations.

\section{Results and discussions}

The simulation procedure commenced with the evaluation of natural frequencies and mode shapes of nanostructures. As an instance, Fig. 3 illustrates six mode shapes of an open-tip nanocone with original and vibrated configurations with the fix-free boundary condition.
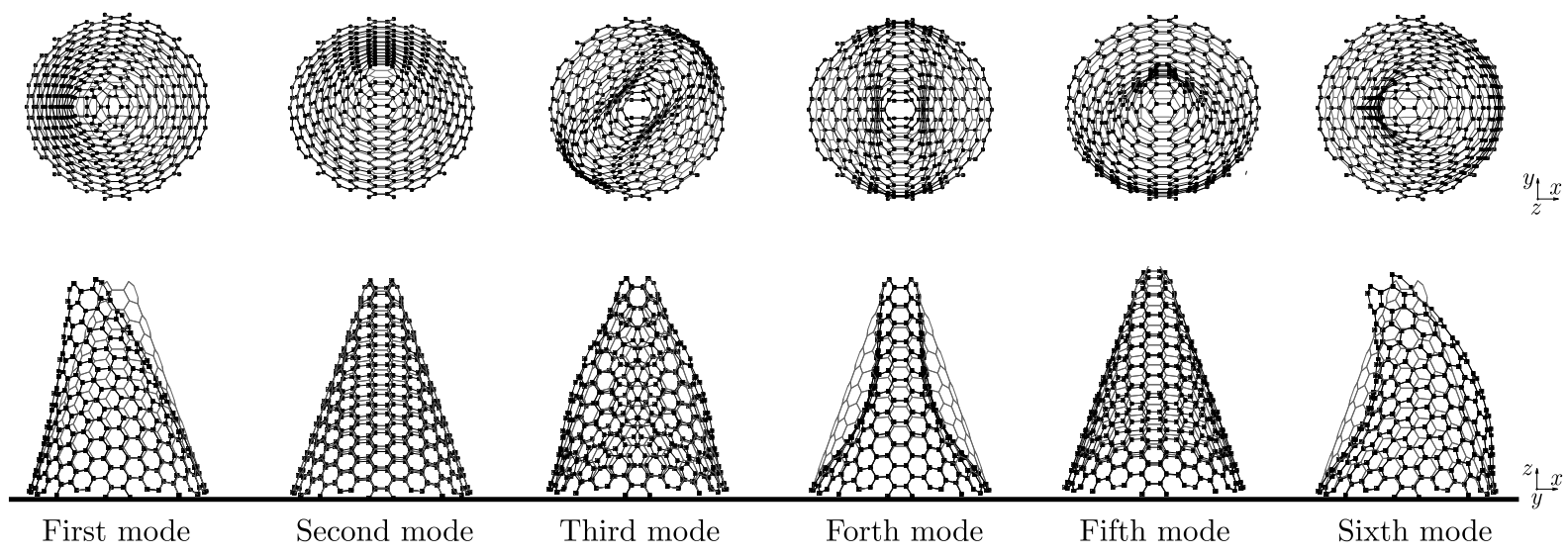

Fig. 3. Six vibrational mode shapes of an open-tip nanocone

Within this simulation, $1 \%$ of four common vacant sites are introduced to the low-dimensional structures and their first natural frequencies are obtained and also compared. Figure 4 shows the overall deviation in the natural frequencies of imperfect homogeneous CNT under the influence of various boundary conditions. Generally, the major change occurred in the fix-fix boundary condition. Nevertheless, the significant deviation happens for the fix-free boundary condition when the lower region is manipulated by impurities where the natural frequency is reduced by nearly $40 \%$ by introducing the pinhole vacancy defect in the structure of the model. The configuration with the free-free boundary condition has a comparatively moderate decline in which the maximum decrease occurs to the model with the pinhole vacancy introduced to the middle of the structure.

Comparing the change in the natural frequencies of one-side capped CNTs, as shown in Fig. 5, it is noted that a significant change occurs in the structure with the fix-free boundary condition. More specifically, the overall change for the fix-free model with single, double, triple, and pinhole vacancy defects is obtained to be approximately $13 \%, 14.5 \%, 16 \%$, and $23 \%$, respectively.

Figure 6 represents the comparative deviation in the natural frequency of two-side capped CNTs under the influence of three different boundary conditions. Based on the computational results, the overall trend of both the free-free and fix-fix boundary conditions are quite similar in spite of a comparatively larger decline for the free-free boundary condition. Due to the symmetric configuration of the model under these two boundary conditions, the vibrational response of the structure under the introduction of defects either in upper and lower sides of the systems is similar. In contrast to the free-free and fix-fix boundary condition, the vibrational response of the two-side capped CNT has a quite different trajectory. While the vacant sites decrease the natural frequency of the model up to maximum $18 \%$ for the impurities introduced to the upper side and middle of the structure, the change is significantly highlighted when the defect is applied 
Atomic defects of homogeneous CNT with free-free boundary condition
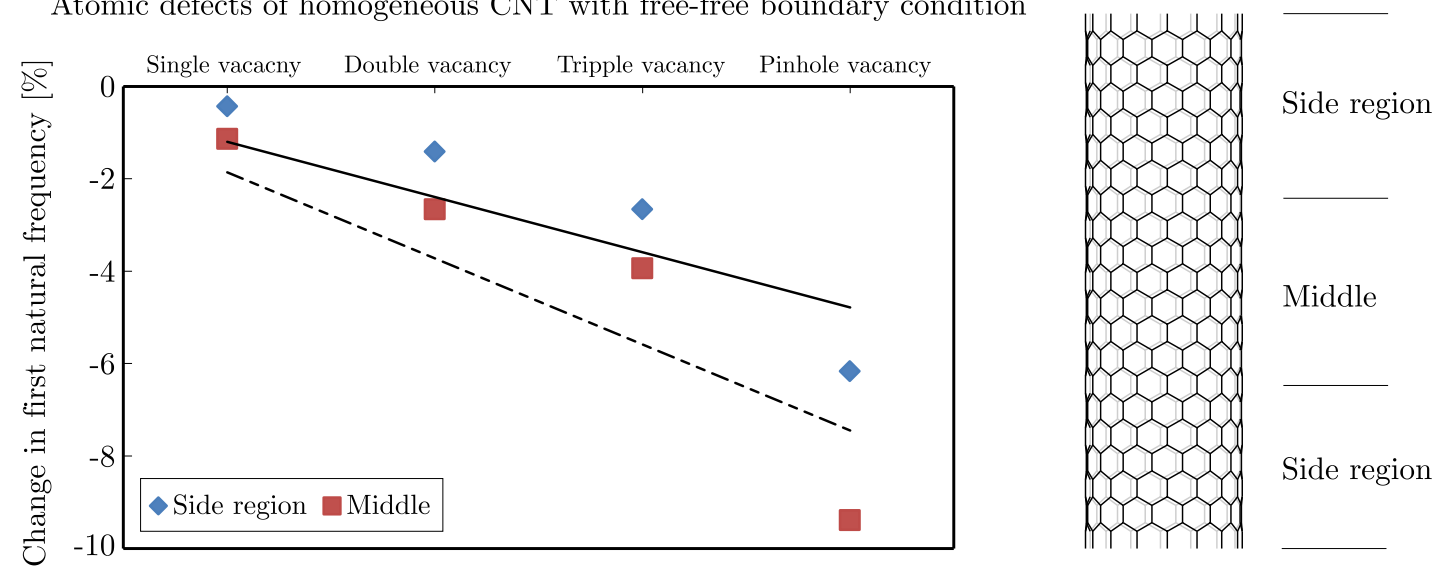

Atomic defects of straight hybrids with fix-free boundary condition
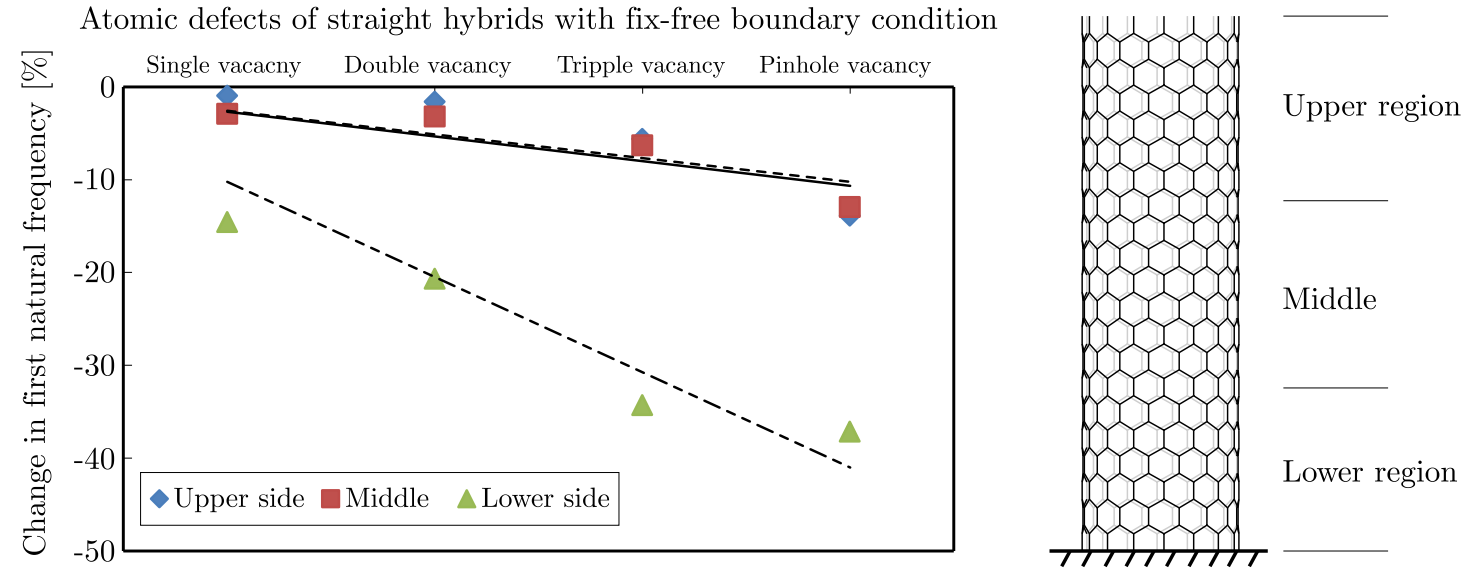

Atomic defects of straight hybrids with fix-fix boundary condition
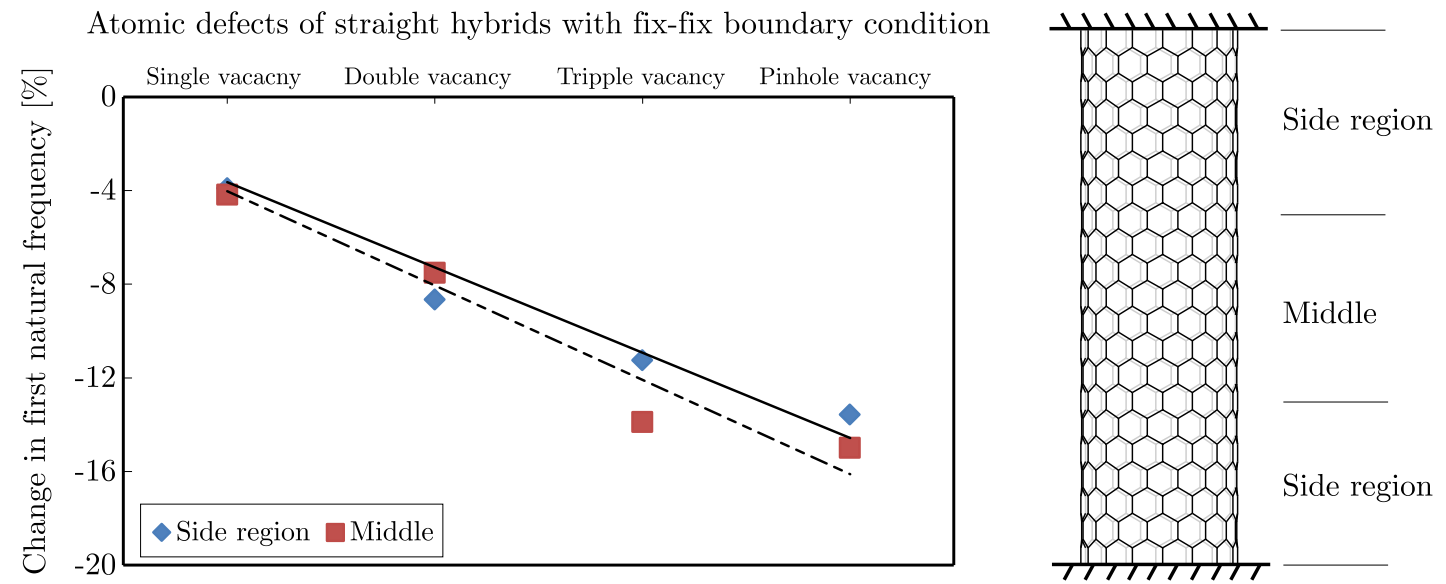

Fig. 4. Change in the natural frequency in $\%$ for a defective homogenous CNT under different boundary conditions

to the lower region of the system where the maximum deviation exceeds $35 \%$ in the case of the pinhole vacancy defect.

The vibrational response of linearly-joined CNTs has been evaluated for different boundary conditions, including free-free, fix-fix, wider tube fix, and thinner tube fix. As shown in Fig. 7, the fundamental frequency of imperfect hybrids reduces significantly, particularly in the case of the thinner tube fix boundary condition. More specifically, the overall change in the natural frequency of straight hybrids is nearly $5 \%$ for a single vacancy defect and reaches nearly $45 \%$ in the worst case which belongs to the thinner tube fix boundary condition. It is noticeable that 
Atomic defects of one-side capped tubes with free-free boundary condition
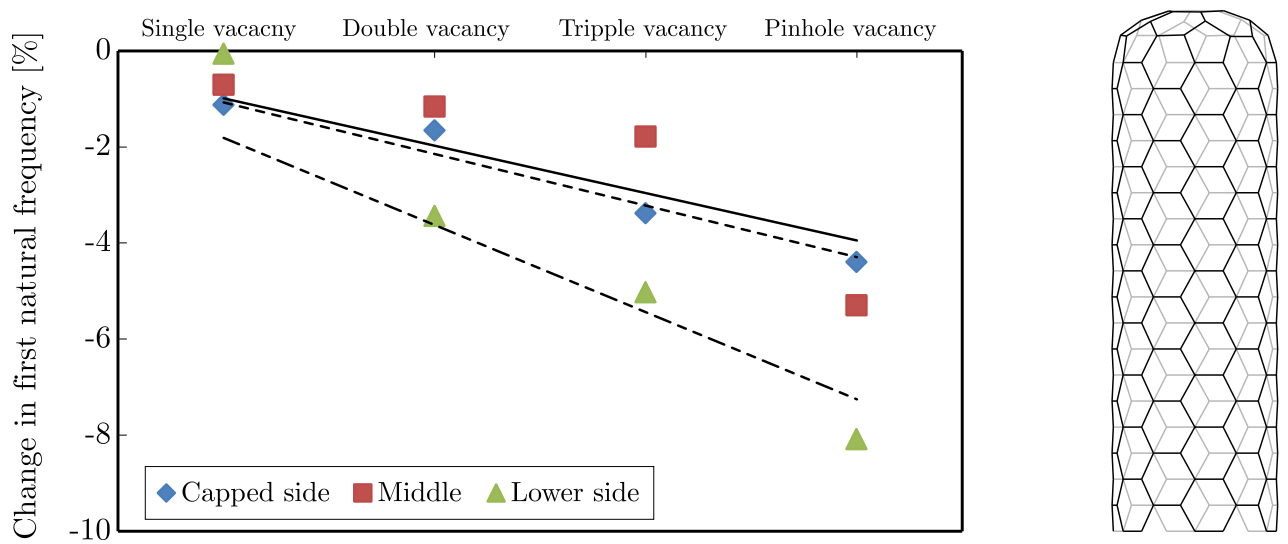

Capped side

Atomic defects of one-side capped tubes with fix-free boundary condition
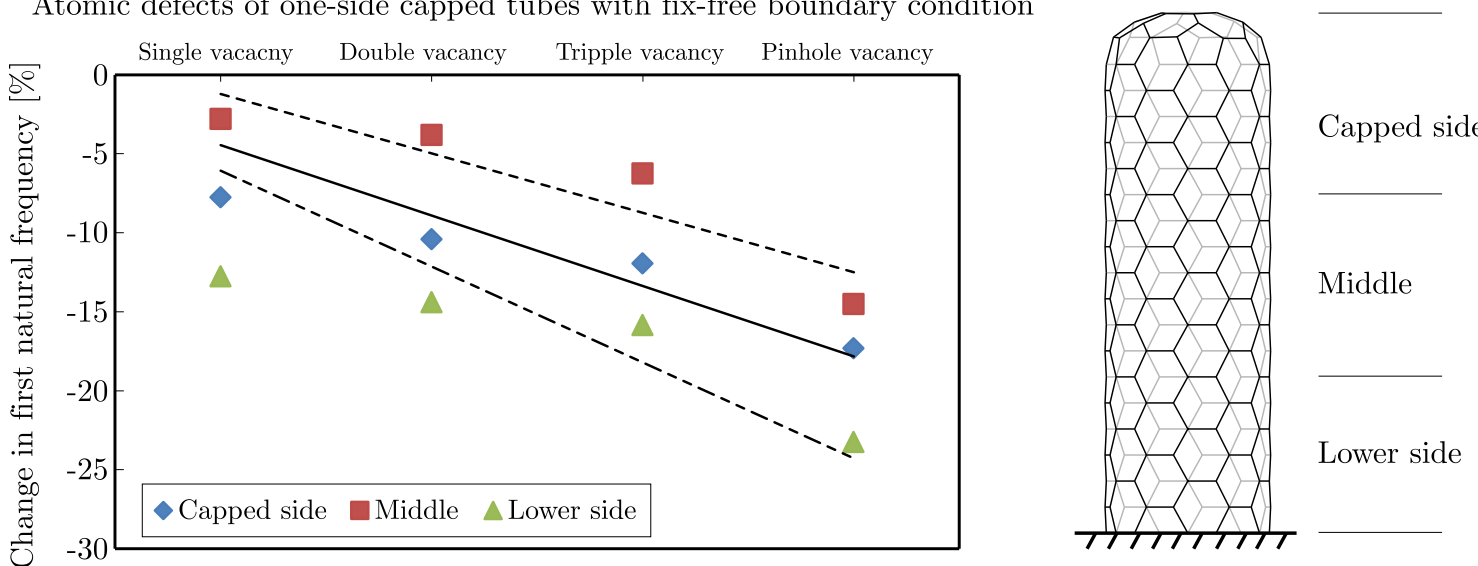

Atomic defects of one-side capped tubes with fix-fix boundary condition
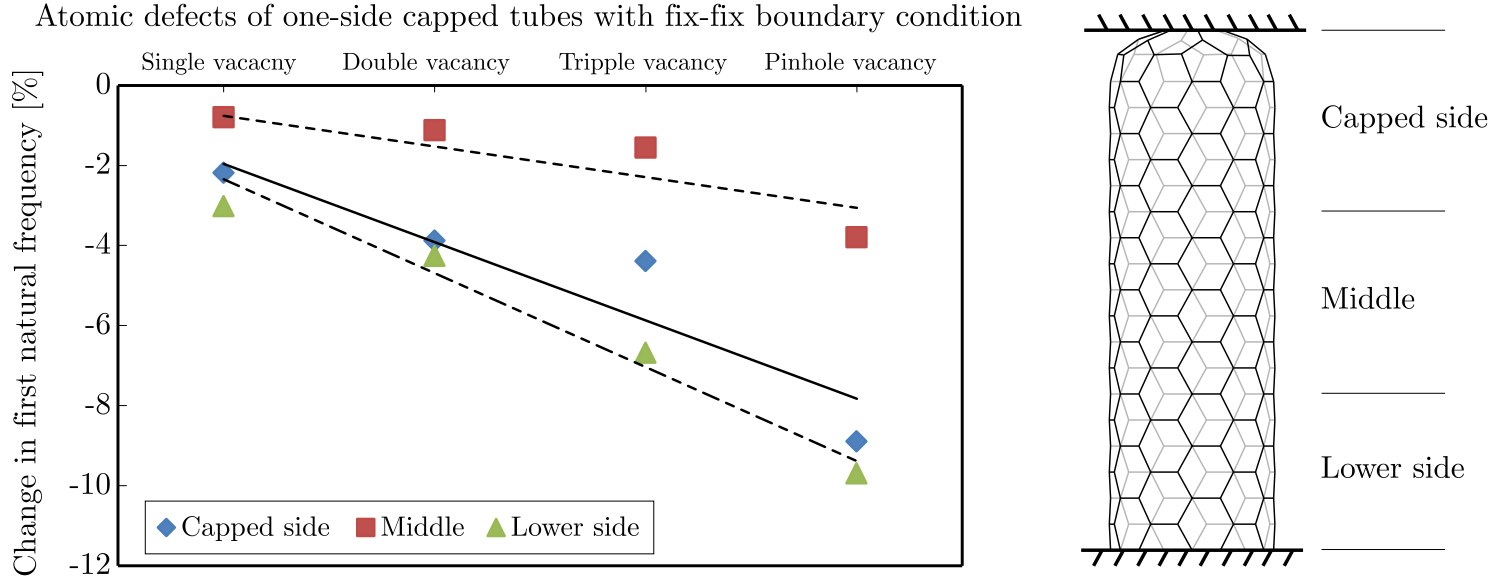

Fig. 5. Change in the natural frequency in $\%$ for a defective one-side capped CNT under different boundary conditions

the thinner fix CNTs reduces the vibrational stability of the structures in comparison with their wider counterparts.

Similarly to straight hybrids, the natural frequencies of imperfect angle-joined CNTs are evaluated under four major boundary conditions, namely free-free, fix-fix, zigzag tube fix, and armchair tube fix. Figure 8 illustrates the overall trend of deviation in the fundamental frequency of the imperfect bending junction. It is noted that the applied impurities on the zigzag tube have a greater influence on the natural frequency of the system in comparison with the armchair 
Atomic defects of two-side capped tubes with free-free boundary condition
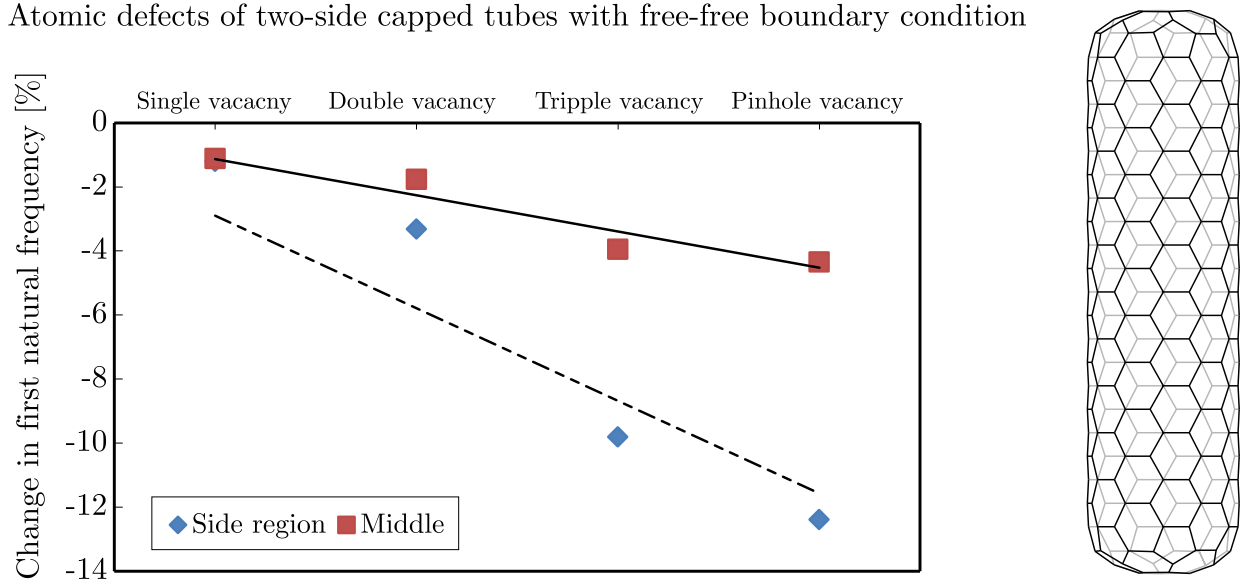

Side region

Atomic defects of two-side capped tubes with fix-free boundary condition

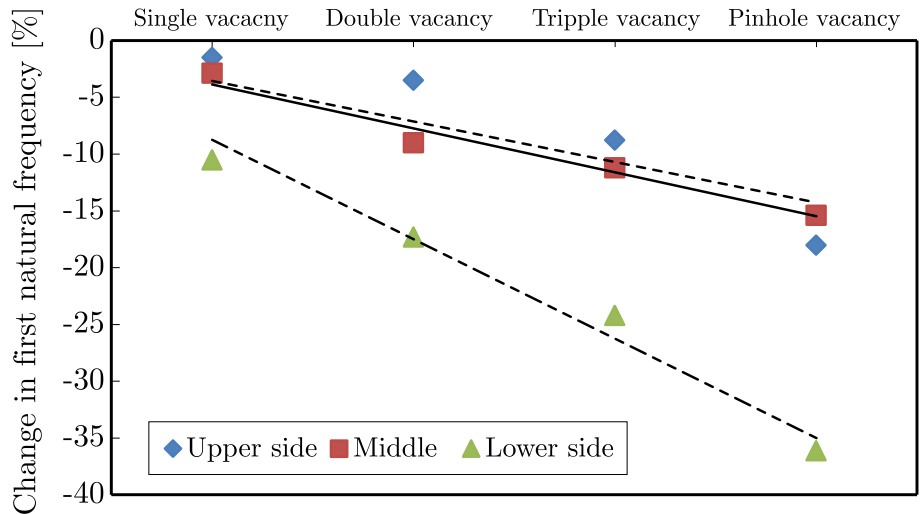

Atomic defects of two-side capped tubes with fix-fix boundary condition
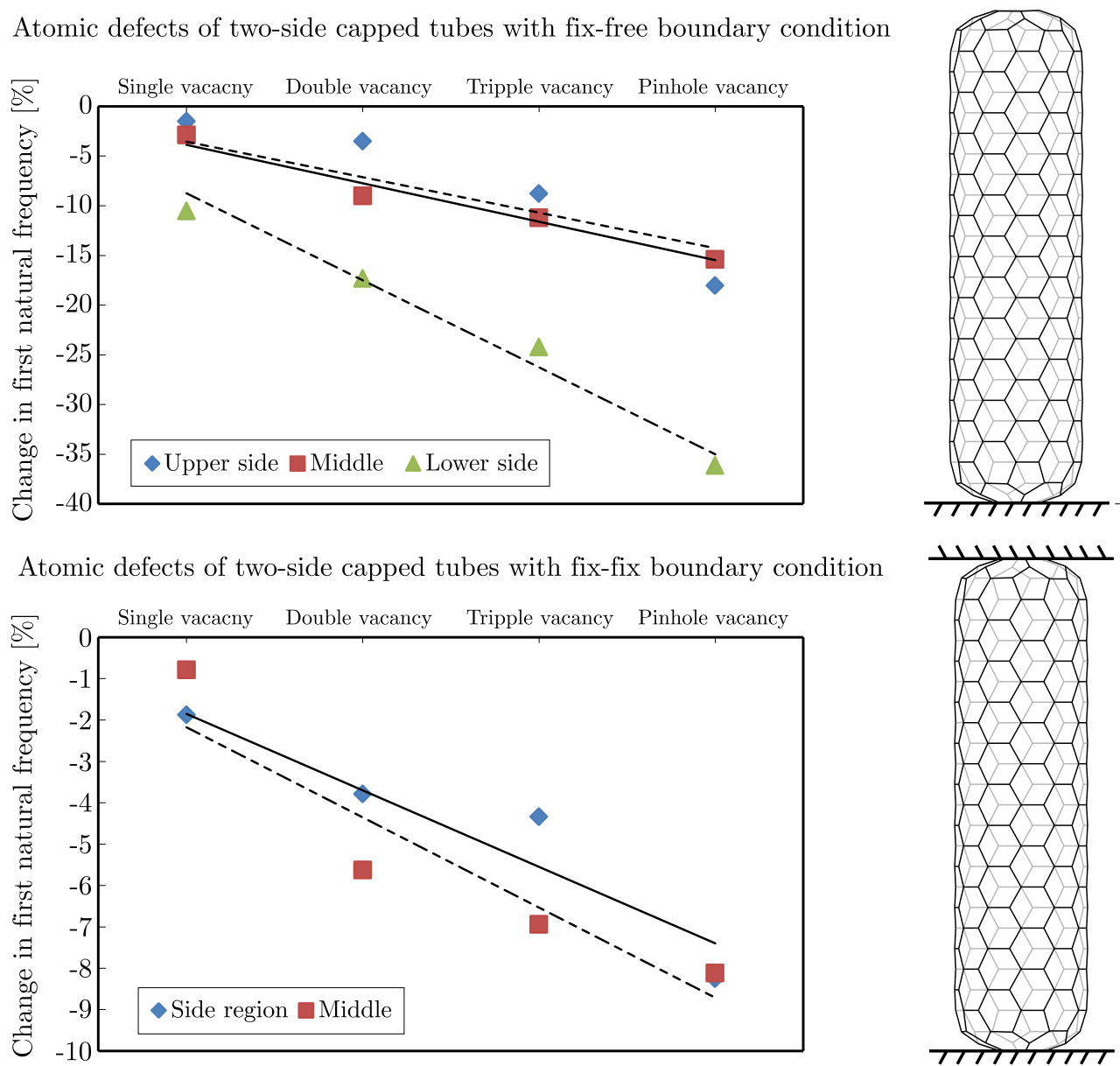

Upper region

Middle

Lower region

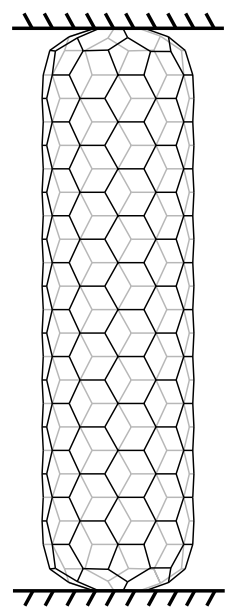

Side region

Middle

Side region

Fig. 6. Change in the natural frequency in $\%$ for a defective two-side capped CNT under different boundary conditions

CNTs. Furthermore, the vibrational stability of the angle-joined CNT under the fix-fix boundary condition is shown to be noticeable. A comparison of the straight and angle-joined hybrids indicates that the bending structures possess a relatively higher strength in vibrational stability.

Based on the results illustrated in Fig. 9, it is noted that the deviation in the natural frequencies of nanocone varies according to the defected region. More specifically, in the case of fix-free boundary conditions, the most highlighted changes in the natural frequency are reported when the atomic defects are introduced to the lower region of the structure. The maximum deviation 

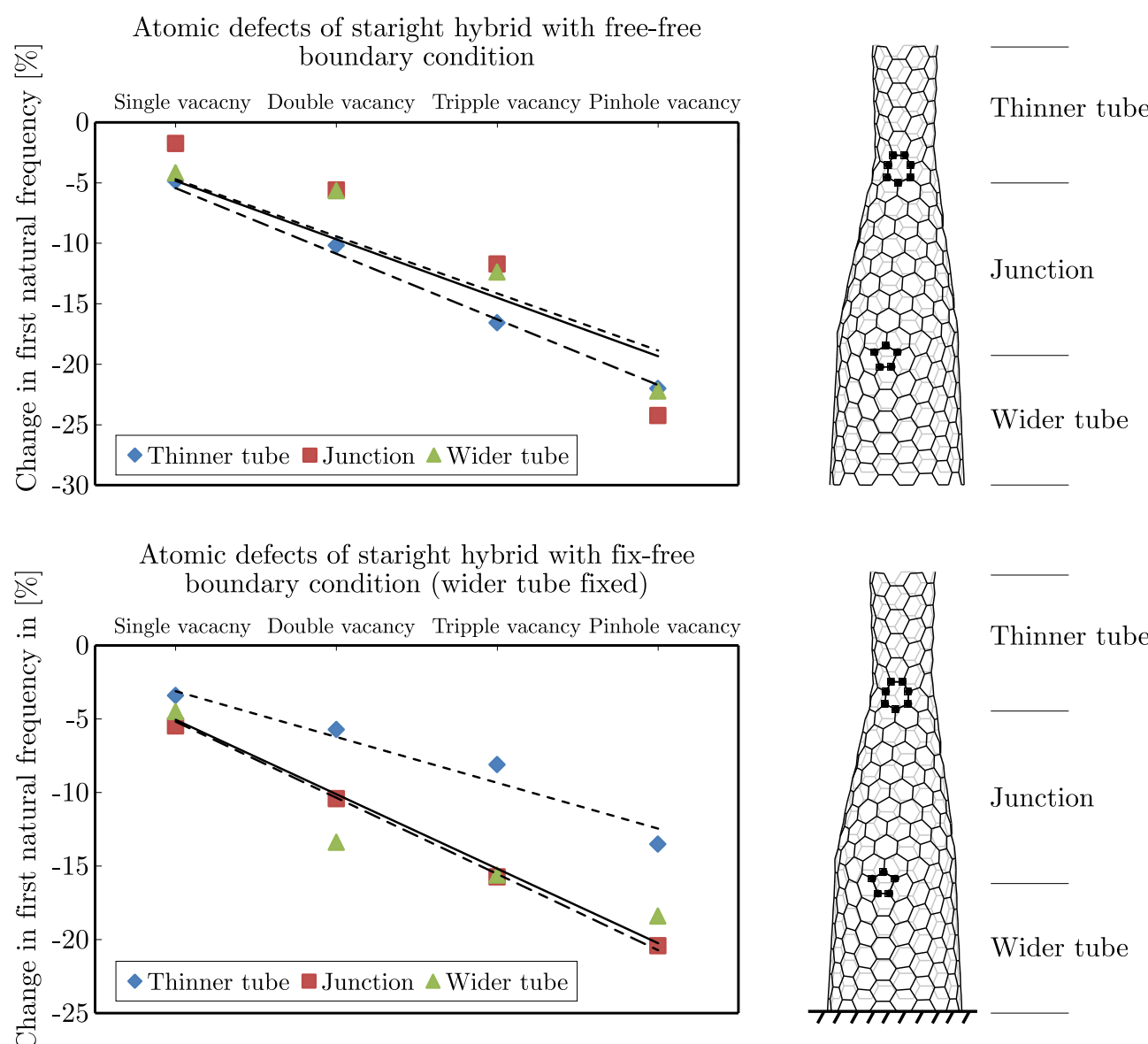

Thinner tube
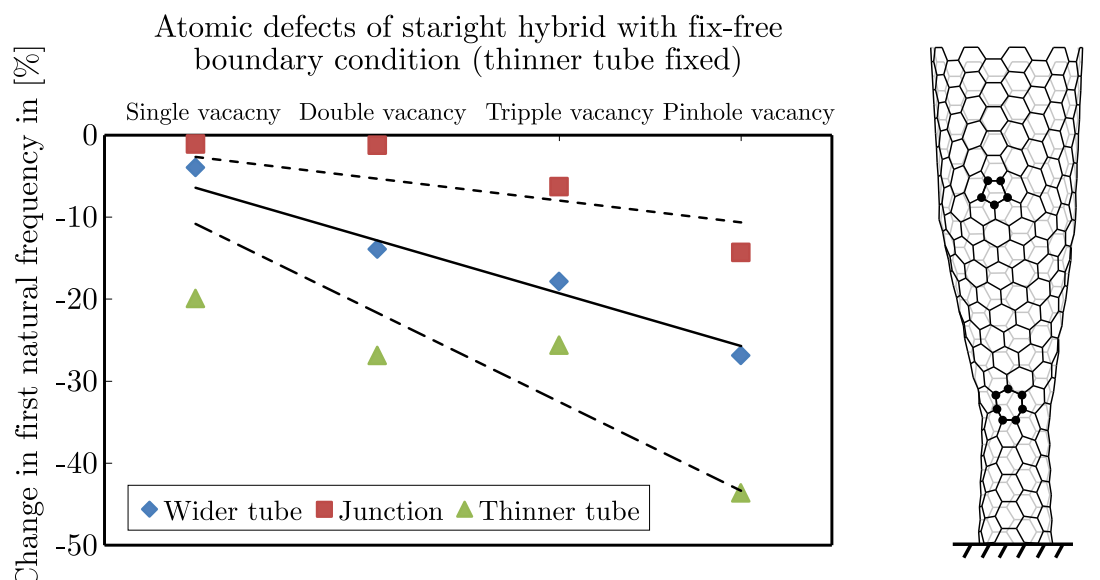

Wider tube

Wider tube
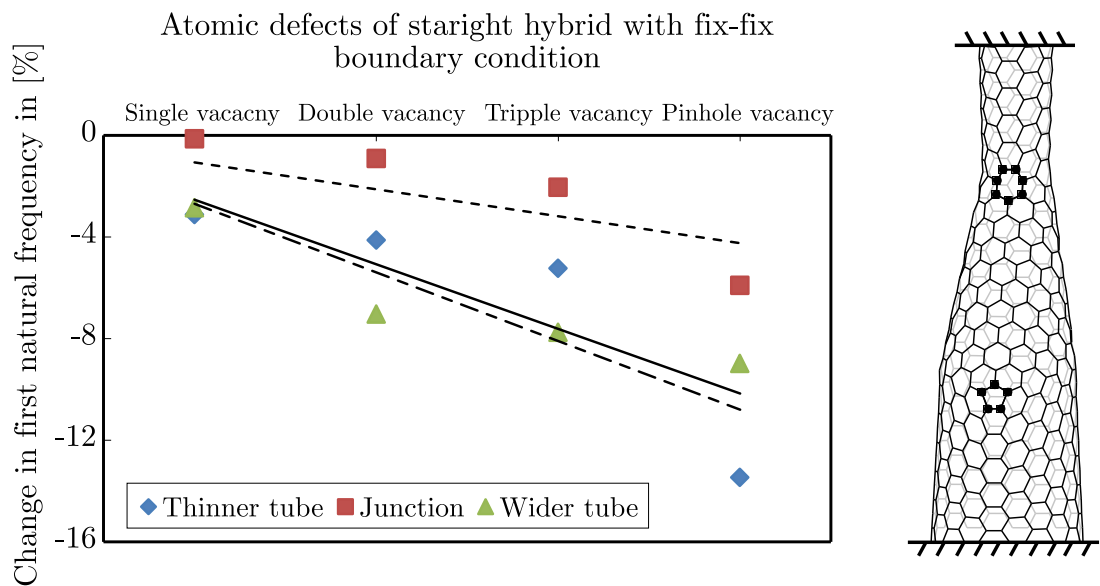

Thinner tube

Junction

Wider tube

Fig. 7. Change in the natural frequency in $\%$ for a defective linearly-joined CNT under different boundary conditions 

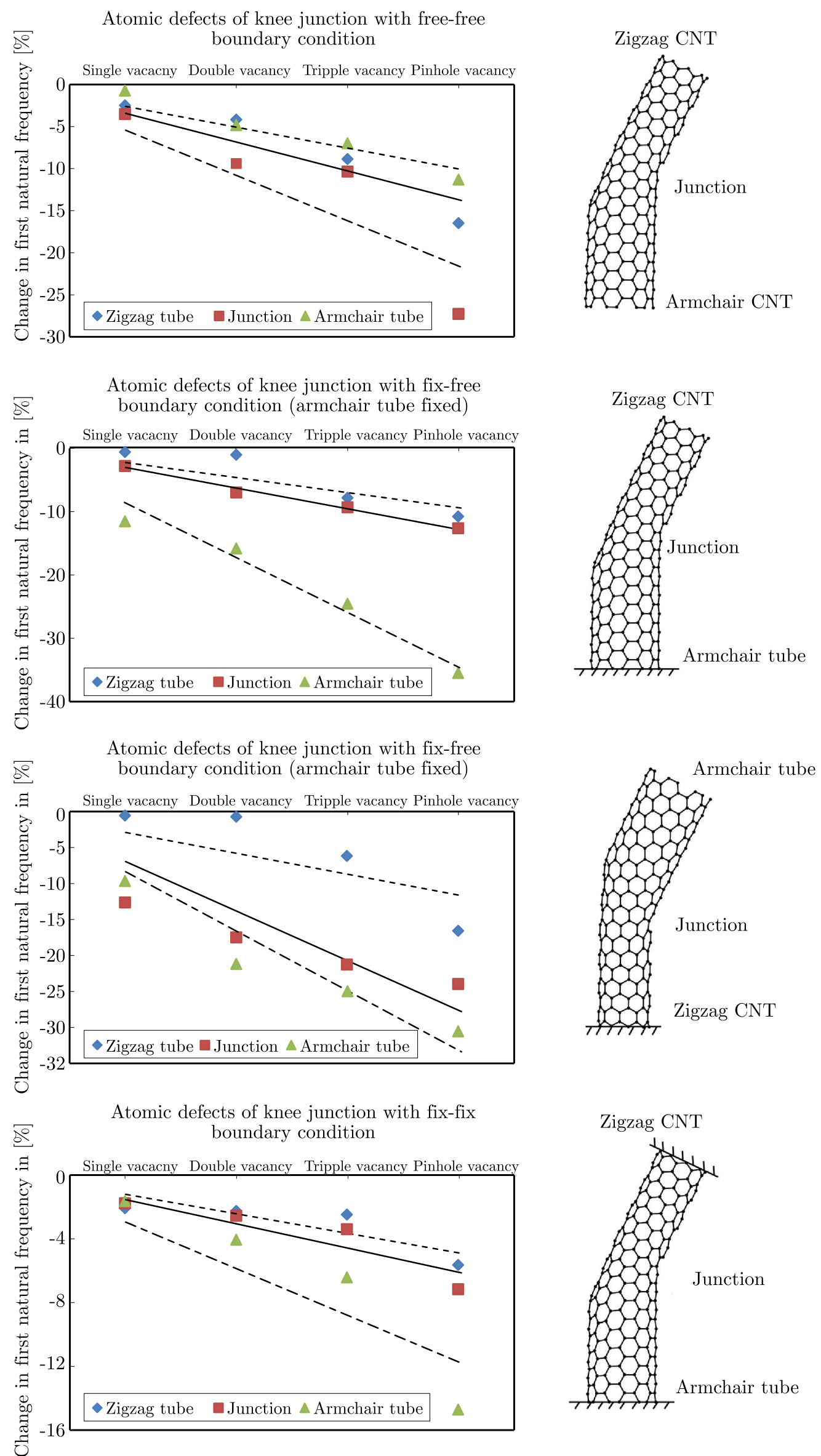

Fig. 8. Change in the natural frequency in \% for a defective angle-joined CNT under different boundary conditions 


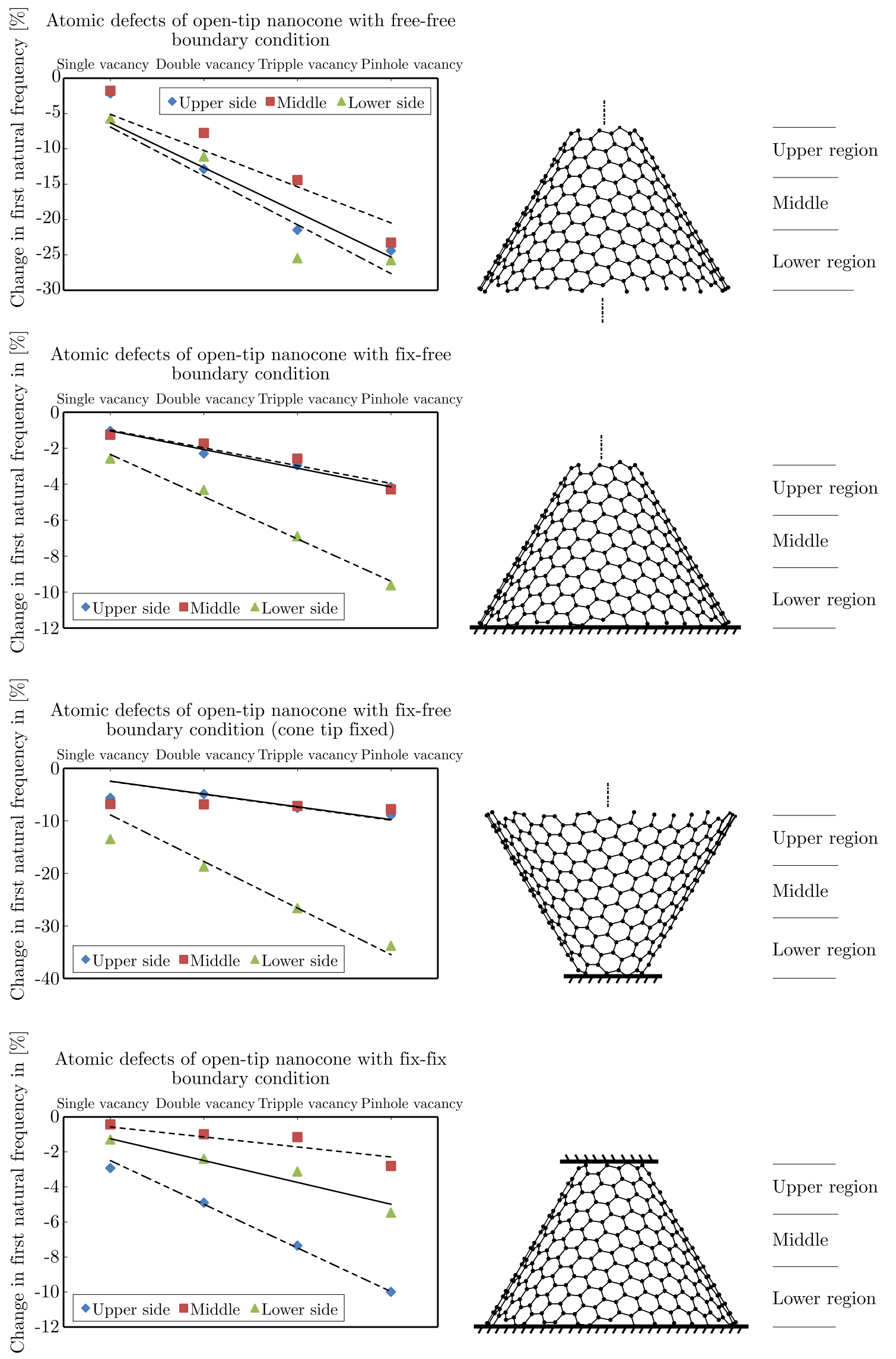

Fig. 9. Change in the natural frequency in $\%$ for a defective open-tip nanocone under different boundary conditions 
observed in the cases of the cone base fix and cone tip fix is reported to be approximately $10 \%$ and $35 \%$, respectively. Apart from some occasional high deviations, the nanocone is noted to be a typical low-dimensional structure which has a significant vibrational stability in comparison with other degenerated nanostructures.

The obtained results from Fig. 10 reveal the fact that the bucky-ball possesses the highest vibrational stability and has the lowest decline in the natural frequency in comparison with the other low-dimensional particles. The change in the natural frequency of the bucky-ball under free-free and fix-fix boundary conditions is reported to be between $1 \%$ up to nearly $9 \%$ in the case of the pinhole vacancy defect.

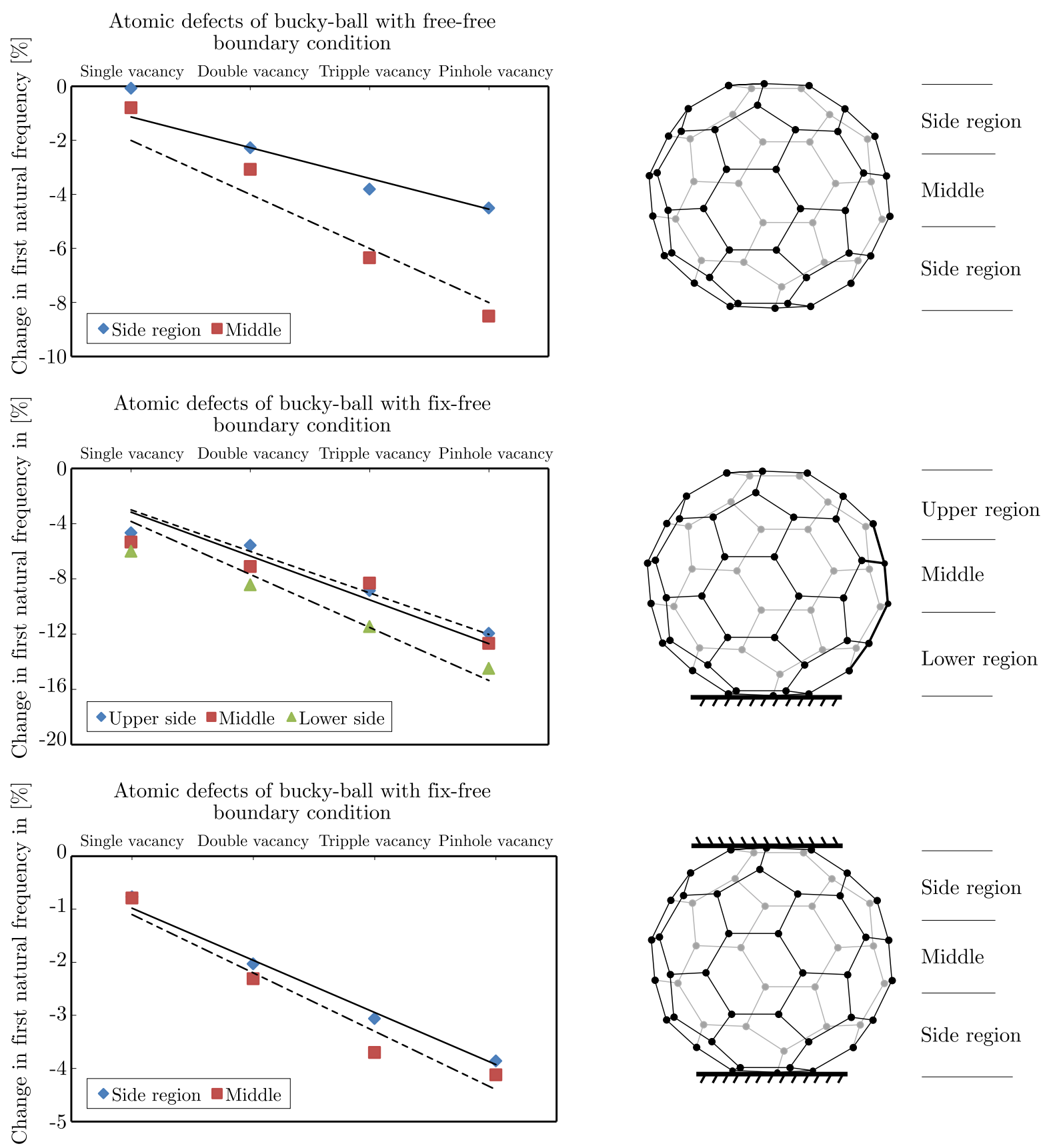

Fig. 10. Change in the natural frequency in $\%$ for a defective bucky-ball under different boundary conditions 


\section{Conclusion}

In this study, several low-dimensional structures including nanocones, linearly- and angle-joined CNTs, bucky-balls, etc. have been simulated in their perfect and defective forms, and their vibrational stability has been evaluated by applying a FE approach. The introduced imperfections are the most common vacant sites, namely mono-, di-, tri-, and pinhole vacancy defects which are applied to the perfect configurations using a custom code in MATLAB. An attempt has been made to evaluate natural frequencies of numerous nanostructures under different boundary conditions. It has been indicated that the natural frequency of the low-dimensional systems decreases with the introduction of atomic modifications. Furthermore, the models have different responses according to their boundary conditions. It is also concluded that bucky-ball and linearly-joined CNT have the least and the most deviations in the natural frequencies, respectively. The significant point of this research is that the applied impurities introduced to the systems have various effects on the fundamental frequencies based on their position in the nanostructures. In addition, it is noted that the angle-joined CNT possesses a higher vibrational stability in comparison with the straight hybrid. Finally, it is concluded that the tube diameter holds a significant influence on the vibrational response of CNTs. The findings of this research opens an avenue for the evaluation of the mechanical property of low-dimensional degenerated systems; nevertheless, much more attention needs to be paid to other methods including density functional theory (DFT), which is the future research objective of the authors.

\section{References}

1. Ardeshana B., Jani U., Patel A., Joahi A.Y., 2017, An approach to modelling and simulation of single-walled carbon nanocones for sensing applications, AIMS Materials Science, 4, 1010-1028

2. Bogush I., Ciobu V., Paladi F., 2017, Advanced computational method for studying molecular vibrations and spectra for symmetrical systems with many degrees of freedom, and its application to fullerene, The European Physical Journal B, 90, 193

3. Chandra N., Namilae S., 2006, Tensile and compressive behavior of carbon nanotubes: effect of functionalization and topological defects, Mechanics of Advanced Materials and Structures, $\mathbf{1 3}$, $115-127$

4. Hollerer S., Celigoj C.C., 2013, Buckling analysis of carbon nanotubes by a mixed atomistic and continuum model, Computational Mechanics, 51, 765-789

5. IIJima S., 1991, Helical microtubules of graphitic carbon, Nature, 354, 56

6. Imani Yengejeh S., Akbar Zadeh M., Ö Chsner A., 2014a, On the buckling behavior of connected carbon nanotubes with parallel longitudinal axes, Applied Physics A, 115, 1335-1344

7. Imani Yengejeh S., Akbar Zadeh M., Öchsner A., 2015a, On the tensile behavior of heterojunction carbon nanotubes, Composites Part B, 75, 274-280

8. Imani Yengejeh S., Kazemi S.A., Öchsner A., 2014b, A numerical evaluation of the influence of atomic modification on the elastic and shear behavior of connected carbon nanotubes with parallel longitudinal axes, Journal of Nano Research, 29, 93-104

9. Imani Yengejeh S., KaZemi S.A., ÖChsner A., 2015b, On the buckling behavior of curved carbon nanotubes, [In:] Mechanical and Materials Engineering of Modern Structure and Component Design, A. Öchsner, H. Altenbach (Eds.), Switzerland, Springer International Publishing, 70, $401-412$

10. Imani Yengejeh S., Kazemi S.A., ÖChsner A., 2016, Advances in mechanical analysis of structurally and atomically modified carbon nanotubes and degenerated nanostructures: A review, Composites Part B: Engineering, 86, 95-107 
11. Imani Yengejeh S., ÖChsner A., 2015, Influence of twisting and distortion on the mechanical properties of carbon nanotubes, Journal of Computational and Theoretical Nanoscience, 12, 443-448

12. KuAng Y.D., He X.Q., 2009, Young's moduli of functionalized single-wall carbon nanotubes under tensile loading, Composites Science and Technology, 69, 169-175

13. Lu J.P., 1997, Elastic properties of carbon nanotubes and nanoropes, Physical Review Letters, 79 , $1297-1300$

14. Mohammadian M., Hosseini S.M., Abolbashari M.H., 2017, Free vibration analysis of dissimilar connected CNTs with atomic imperfections and different locations of connecting region, Physica B: Condensed Matter, 524, 34-46

15. Mylvaganam K., Vodenitcharova T., Zhang L.C., 2006, The bending-kinking analysis of a single-walled carbon nanotube - a combined molecular dynamics and continuum mechanics technique, Journal of Materials Science, 41, 3341-3347

16. Ruoff R.S., Lorents D.C., 1995, Mechanical and thermal properties of carbon nanotubes, Carbon, 33, 925-930

17. Tserpes K.I., Papanikos P., 2007, The effect of Stone-Wales defect on the tensile behavior and fracture of single-walled carbon nanotubes, Composite Structures, 79, 581-589

18. YaO X.-H., Han Q., Xin H., 2008, Bending buckling behaviors of single- and multi-walled carbon nanotubes, Computational Materials Science, 43, 579-590 